\title{
Competências do enfermeiro em emergência e o produto do cuidar em enfermagem: revisão integrativa
}

\author{
Competences of the emergency nurse and the product of nursing care: an integrative review
}

Como citar este artigo:

Jesus JA, Balsanelli AP. Competences of the emergency nurse and the product of nursing care: an integrative review. Rev Rene. 2020;21:e43495. DOI: https://doi.org/10.15253/2175-6783.20202143495

DJucinei Araújo de Jesus ${ }^{1}$

(D)Alexandre Pazetto Balsanelli ${ }^{1}$

${ }^{1}$ Universidade Federal de São Paulo.

São Paulo, SP, Brasil.

\author{
Autor correspondente: \\ Jucinei Araújo de Jesus \\ Estrada de Itapecerica, 1187, Bl 10 Apto 44 \\ Vila das Belezas, CEP: 05835-003. \\ São Paulo, SP, Brasil. \\ E-mail: jucinei.araujo06@huhsp.org.br
}

\begin{abstract}
RESUMO
Objetivo: identificar as competências do enfermeiro em emergência e o produto do cuidar em enfermagem. Métodos: revisão integrativa com 16 estudos incluídos, identificados por meio de busca eletrônica na Literatura Latino-Americana e do Caribe em Ciências da Saúde, Sistema Online de Busca e Análise de Literatura Médica, Cumulative Index to Nursing and Allied Health Literature e Scientific Eletronic Library Online a partir de descritores: Professional Competence, Emergency Nursing, Nursing Assessment, Process Assessment (Health Care), Nursing Service, Hospital, Nursing Care e Professional Practice. Resultados: foram incluídos 16 estudos, dos quais 12 apresentaram as competências mínimas requeridas ao enfermeiro de emergência, e 4 se referiam ao produto do cuidar em enfermagem. Conclusão: as competências que mais emergiram foram "desempenho assistencial" e "liderança". 0 produto do cuidado em enfermagem precisa ser mais explorado, sobretudo nas emergências.
\end{abstract}

Descritores: Competência Profissional; Cuidados de Enfermagem; Enfermagem em Emergência; Prática Profissional.

\footnotetext{
ABSTRACT

Objective: identifying the competences of the emergency nurse and the product of nursing care. Methods: integrative review of 16 studies identified through an electronic search in the databases Literatura Latino-Americana e do Caribe em Ciências da Saúde, Sistema Online de Busca e Análise de Literatura Médica, Cumulative Index to Nursing and Allied Health Literature, and Scientific Eletronic Library Online, using the descriptors: Professional Competence, Emergency Nursing, Nursing Assessment, Process Assessment (Health Care), Nursing Service, Hospital, Nursing Care and Professional Practice. Results: 16 studies were included. 12 presented the minimum competences required from the emergency nurse and 4 referred to the product of nursing care. Conclusion: the most commonly present competences were "performance of the attention" and "leadership". The product of nursing care needs to be further explored, especially in emergencies.

Descriptors: Professional Competence; Nursing Care; Emergency Nursing; Professional Practice.
} 


\section{Introdução}

Nos serviços de emergência, os enfermeiros estão envolvidos no gerenciamento do cuidado e em ações gerenciais articuladas ${ }^{(1)}$. 0 trabalho desses profissionais tem sido notório em consequência do avanço de sua atuação, seja pelos saberes ou práticas, seja pelo espaço que ocupam na produção de cuidados e na organização do serviço. Isso se deve às ações de enfermagem estarem inter-relacionadas e à marcante presença do conhecimento técnico-científico ${ }^{(2)}$.

A responsabilidade pela gestão do cuidado compete ao enfermeiro. Este deve avaliar o produto gerado que refletirá nos serviços prestados, com a finalidade de propor novas intervenções ${ }^{(3)}$. Isso porque o desafio primordial é apropriar-se da ciência e da complexidade, inerentes ao produto do cuidado, com a finalidade de integrá-las ao modelo tradicional de gestão. Por sua vez, tal integração resultará em melhor compreensão da equipe, do ambiente e do comportamento organizacional, com possibilidades de projetar inovações na maneira de investigar e liderar ${ }^{(4)}$.

O capital humano e serviços de suporte entendidos como fatores estruturais, bem como o método de organização do trabalho pautado pelo planejamento assistencial, atenção ao paciente/família e interação multidisciplinar, são fatores estruturais que definem o produto do cuidar em enfermagem, e tal coesão acarretará melhor avaliação do produto ${ }^{(5)}$.

Para que tal avaliação seja superior, o enfermeiro de emergência necessita de um conjunto de competências para desempenhar bem a sua função. Estudo recente ${ }^{(6)}$ identificou-as por meio de uma matriz, destacando-se: desempenho assistencial, trabalho em equipe, liderança, humanização, relacionamento interpessoal, tomada de decisão, direcionamento para resultados e proatividade. A partir desse resultado ${ }^{(6)}$, torna-se importante tanto identificar como essas competências têm sido estudadas com os enfermeiros que atuam no serviço de emergência quanto explorar o conceito do produto do cuidado disponível na literatura.
Dessa forma, neste estudo, objetivou-se identificar as competências do enfermeiro em emergência e o produto do cuidar em enfermagem.

\section{Métodos}

Trata-se de uma revisão integrativa da literatura $^{(7)}$ que obedeceu às seguintes etapas: formulação do problema, coleta de dados, avaliação, análise e interpretação, apresentação dos resultados e conclusões ${ }^{(7-8)}$. Utilizou-se ainda a estratégia $\mathrm{PICo}^{(9)}$ na seguinte representação: P: enfermeiro, I: competências e o produto do cuidado, Co: serviço de emergência. Assim, a pergunta de pesquisa foi: "Quais são as competências do enfermeiro em emergência e o produto do cuidar em enfermagem?"

O levantamento bibliográfico foi realizado de julho a setembro de 2019, por meio da busca eletrônica na Literatura Latino-americana e do Caribe em Ciências da Saúde (LILACS), Sistema Online de Busca e Análise de Literatura Médica (MEDLINE/PubMed), Cumulative Index to Nursing and Allied Health Literature (CINAHL) e Scientific Eletronic Library Online (SciELO), a partir de descritores contemplados nos Descritores em Ciências da Saúde (DeCS) e no MeSH (Medical Subject Headings): Professional Competence, Emergency Nursing, Nursing Assessment, Process Assessment (Health Care), Nursing Service, Hospital, Nursing Care e Professional Practice.

Realizou-se o cruzamento entre os descritores utilizando o operador booleano "and" como combinação restritiva, adotando-se as seguintes estratégias: 1) Professional Competence and Emergency Nursing; 2) Professional Competence and Nursing Assessment and Emergency Nursing; 3) Process Assessment (Health Care) and Nursing Service, Hospital and Nursing Care; e 4) Professional Competence and Professional Practice and Emergency Nursing.

Os critérios de inclusão foram: artigos originais publicados entre os anos de 2014 e 2019, disponíveis na íntegra, em português, inglês e espanhol. Esse período foi escolhido considerando a publicação do es- 
tudo sobre Matriz de Competências em Enfermagem na Emergência ${ }^{(6)}$, de 2014, e o conceito do produto do cuidado, publicado em $2015^{(10)}$. Os critérios de exclusão foram: teses, dissertações, resenhas, editoriais e resumos de anais de congresso.

Com base na combinação dos descritores, foram localizadas 416 produções. Procedeu-se às análises manuais das publicações pelos títulos e resumos; e ao posterior descarte de repetições - neste caso, três artigos. Diante disso, excluíram-se 397 artigos por não coadunarem com a pergunta de pesquisa estabelecida inicialmente. A amostra final constituiu-se de 16 artigos.

Os artigos selecionados foram analisados na íntegra e classificados em dois grupos: produto do cuidar em enfermagem; e competências do enfermeiro em emergência. A análise e interpretação dos dados foram realizadas de forma organizada, elencando os seguintes itens: nome do(s) autor(es), periódico, ano, objetivos e principais resultados.

Foi realizada a classificação do nível de evidências dos estudos mediante os critérios hierárquicos elencados pela Agency for Healthcare Research and Quality (AHRQ), dos Estados Unidos da América, cujas gradações estão distribuídas em seis níveis: o nível 1 é utilizado para metanálise de múltiplos estudos controlados; o nível 2, para estudo individual com desenho experimental; o nível 3, para estudo quase experimental ou caso-controle; o nível 4, para estudos não experimentais; o nível 5, para relatórios de casos de qualidade verificável ou avaliação de programas; e o nível 6, para opinião de autoridades, de comitês de especialistas e interpretações de informações não baseadas em pesquisa ${ }^{(11)}$.

As competências profissionais destacadas na matriz de competência profissional do enfermeiro em emergências ${ }^{(6)}$ foram identificadas nos artigos de forma descritiva ou implícita a partir das definições elencadas.

O processo de seleção dos artigos está descrito a seguir, o qual se apresenta num diagrama de fluxo de seleção e identificação dos estudos de acordo com o Preferred Reporting Items for Systematic Reviews and Meta-Analyses (PRISMA) ${ }^{(12)}$ (Figura 1).

Participaram das etapas descritas no PRISMA dois pesquisadores com experiência nessa temática, e não houve divergência entre eles.
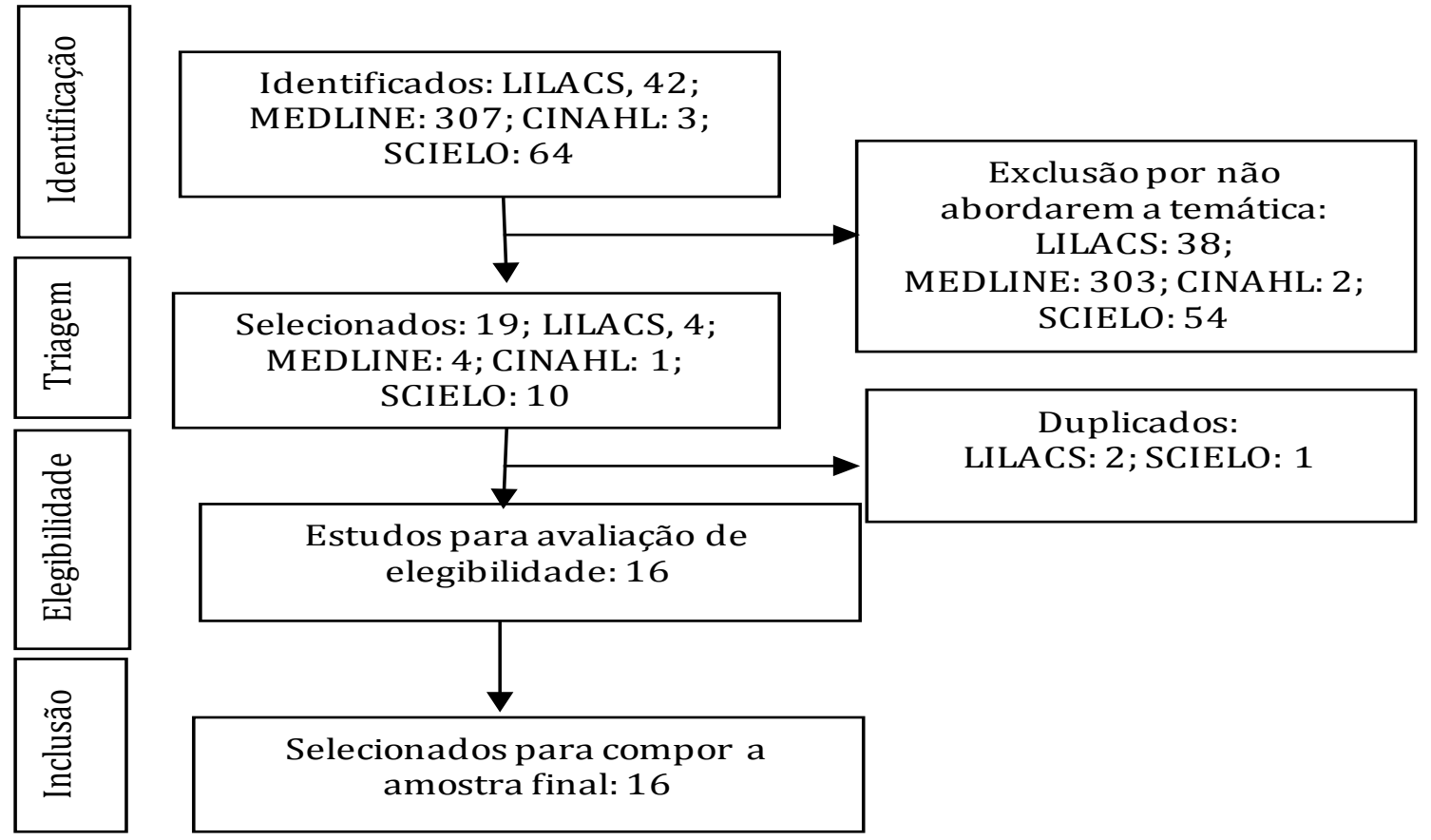

Figura 1 - Fluxograma de seleção das publicações - PRISMA ${ }^{(12)}$. São Paulo, SP, Brasil, 2019 


\section{Resultados}

Dos 16 artigos inclusos nesta revisão, 4 estudos $^{(10,13-15)}$ abordaram o produto do cuidar em enfermagem, e $12^{(16-27)}$ apresentaram as competências mínimas requeridas ao enfermeiro que atua em emergências.

Os artigos que abordaram o produto do cuidar em enfermagem foram desenvolvidos e publicados no Brasil, entre os anos de 2015 e 2018 (10,13-15) $^{\text {, e tiveram }}$ em sua população enfermeiros clínicos. Foram encontrados apenas quatro artigos na perspectiva da temática; três deles versam sobre a criação e validação do construto, e o quarto, sobre sua aplicabilidade em hospitais previamente selecionados. A Figura 2 descreve os objetivos, os principais resultados encontrados e o nível de evidência.

Doze estudos apresentaram as competências requeridas ao enfermeiro que atua em emergências de acordo com a matriz publicada em 2015 (27). 0 de-

\begin{tabular}{|c|c|c|c|}
\hline $\begin{array}{l}\text { Autor/Periódico/ } \\
\text { Ano }\end{array}$ & Objetivos & Principais resultados & $\begin{array}{c}\text { Nível de } \\
\text { evidência }\end{array}$ \\
\hline $\begin{array}{l}\text { Cucolo DF, Perroca } \\
\mathrm{MG}^{(10)} / \text { Acta Paul } \\
\text { Enferm/2015 }\end{array}$ & $\begin{array}{l}\text { Identificar os principais fatores } \\
\text { intervenientes na produção do cuidado } \\
\text { em enfermagem para geração de itens de } \\
\text { instrumento }\end{array}$ & $\begin{array}{l}\text { Categorias temáticas: Planejamento, intervenção e } \\
\text { avaliação do cuidado; Dimensionamento e qualificação } \\
\text { da equipe de enfermagem; Recursos necessários para } \\
\text { prestar assistência; e Interação multiprofissional }\end{array}$ & 4 \\
\hline $\begin{array}{l}\text { Cucolo DF, Perroca } \\
\text { MG }^{(13)} / \text { Rev Latino-Am } \\
\text { Enfermagem } / 2015\end{array}$ & $\begin{array}{l}\text { Desenvolver e validar o conteúdo de um } \\
\text { instrumento sobre produção do cuidado de } \\
\text { enfermagem }\end{array}$ & $\begin{array}{l}\text { Fase 1: geração de itens e desenvolvimento do } \\
\text { instrumento; Fase 2: teste do protótipo; Fase 3: } \\
\text { validação do conteúdo }\end{array}$ & 4 \\
\hline $\begin{array}{l}\text { Cucolo DF, Perroca } \\
\mathrm{MG}^{(14)} / \text { Rev Latino-Am } \\
\text { Enfermagem } / 2017\end{array}$ & $\begin{array}{l}\text { Verificar as estimativas de confiabilidade e } \\
\text { validade de construto da escala Avaliação } \\
\text { do Produto do Cuidar em Enfermagem } \\
\text { (APROCENF) e sua aplicabilidade }\end{array}$ & $\begin{array}{l}\text { Realizadas } 172 \text { avaliações em unidades de internação } \\
\text { de clínica médica e cirúrgica, Pediátrica, Materno } \\
\text { Infantil, Unidade de Terapia Intensiva Geral, Unidade } \\
\text { de Terapia Intensiva Coronária e Unidade de Terapia } \\
\text { Intensiva Pediátrica; Classificação do produto do cuidar } \\
\text { em enfermagem: ruim (3; 1,8\%), regular (38; 22,1\%), } \\
\text { bom }(111 ; 64,5 \%) \text { e ótimo }(20 ; 11,6 \%)\end{array}$ & 4 \\
\hline $\begin{array}{l}\text { Chotolli MR, et } \\
\text { al }^{(15)} / \text { Rev Bras } \\
\text { Enferm/2018 }\end{array}$ & $\begin{array}{l}\text { Avaliar o produto do cuidar em unidades de } \\
\text { hospitais especializados, identificando os } \\
\text { itens que mais contribuíram para o escore; } \\
\text { comparar o produto do cuidar entre os } \\
\text { hospitais, unidades e turnos; e verificar a } \\
\text { associação entre as variáveis demográficas } \\
\text { e profissionais e o produto do cuidar }\end{array}$ & $\begin{array}{l}\text { Nos hospitais avaliados, o produto entregue pela } \\
\text { enfermagem mostrou-se "bom" e evidenciou que o } \\
\text { APROCENF é uma ferramenta importante subsidiando } \\
\text { os enfermeiros na tomada de decisão e gestão do } \\
\text { cuidado. }\end{array}$ & 4 \\
\hline
\end{tabular}

Figura 2 - Sumário das características dos estudos do produto do cuidar em enfermagem. São Paulo, SP, Brasil, 2019 -sempenho assistencial foi identificado em 4 (33,3\%) e a liderança em $5(41,7 \%)$ estudos. Nota-se a preocupação dos estudos em mapear essas competências tes resultados são apresentados na Figura 3.

Os estudos identificados na Figura 3 evidenciaram as competências destacadas na matriz de competências $^{(6)}$, confirmando sua autenticidade e aplicabilidade nas emergências, o que possibilitará desenvolvimento do perfil profissional aos enfermeiros.

O nível de evidências dos 16 artigos selecionados demonstrou sua relevância uma vez que atenderam às proposituras estabelecidas nessa revisão mediante o emprego do $\mathrm{AHRQ}^{(11)}$ ao alcançarem níveis entre 4 e 5 . Destaca-se a necessidade de avançar em desenhos metodológicos que produzam níveis de evidências mais robustos para fundamentar a prática gerencial do enfermeiro. como necessárias para o trabalho do enfermeiro. Es- 


\begin{tabular}{|c|c|c|c|}
\hline Autor/Periódico/Ano & Objetivos & Competências Identificadas & $\begin{array}{l}\text { Nível de } \\
\text { Evidência }\end{array}$ \\
\hline $\begin{array}{l}\text { Rawson } \mathrm{H} \text {, et } \mathrm{al}^{(16)} / \mathrm{J} \\
\text { Australas Emerg Nurs } \\
\mathrm{J} / 2017\end{array}$ & $\begin{array}{l}\text { Conhecimento dos enfermeiros e dos cuidados de pacientes } \\
\text { idosos na emergência }\end{array}$ & $\begin{array}{l}\text { Desempenho assistencial, } \\
\text { humanização }\end{array}$ & 5 \\
\hline $\begin{array}{l}\text { Martínez-Segura E, et al }{ }^{(17)} / \\
\text { Emergencias } / 2017\end{array}$ & $\begin{array}{l}\text { Identificar perfil sociodemográfico e níveis de competências } \\
\text { de enfermeiros nas emergências }\end{array}$ & $\begin{array}{l}\text { Tomada de decisão, } \\
\text { Proatividade }\end{array}$ & 4 \\
\hline $\begin{array}{l}\text { Jenkins } \mathrm{J}^{(18)} / \text { Emerg } \\
\text { Nurse/2016 }\end{array}$ & $\begin{array}{l}\text { Identificar habilidades clínicas nas práticas baseadas em } \\
\text { evidências nas emergências }\end{array}$ & $\begin{array}{c}\text { Trabalho em equipe, } \\
\text { Direcionamento para resultados }\end{array}$ & 5 \\
\hline $\begin{array}{l}\text { Burchill CN, Polomano R }{ }^{(19)} / \\
\text { Int Emerg Nurs/2016 }\end{array}$ & $\begin{array}{l}\text { Compreender a relação entre atitudes e fatores demográficos } \\
\text { dos enfermeiros e as práticas baseadas em evidências }\end{array}$ & $\begin{array}{c}\text { Proatividade, } \\
\text { Direcionamento para resultados }\end{array}$ & 5 \\
\hline $\begin{array}{l}\text { Cabilan CJ, et al }{ }^{(20)} / \mathrm{J} \text { Adv } \\
\text { Nurs } / 2016\end{array}$ & $\begin{array}{l}\text { Avaliar o conhecimento dos enfermeiros sobre medicação } \\
\text { nas emergências }\end{array}$ & $\begin{array}{c}\text { Tomada de decisão, } \\
\text { Direcionamento para resultados }\end{array}$ & 4 \\
\hline $\begin{array}{l}\text { Mello JJ, et al }{ }^{(21)} / \text { Int Emerg } \\
\text { Nurs/2016 }\end{array}$ & $\begin{array}{l}\text { Avaliar a competência dos enfermeiros nas emergências } \\
\text { psiquiátricas }\end{array}$ & Desempenho assistencial & 5 \\
\hline $\begin{array}{l}\text { Thomas D, et al }{ }^{(22)} / J \text { Emerg } \\
\text { Nurs } / 2015\end{array}$ & $\begin{array}{l}\text { Descrever os protocolos utilizados na triagem para manejo } \\
\text { da dor }\end{array}$ & $\begin{array}{l}\text { Desempenho assistencial, } \\
\text { Liderança }\end{array}$ & 5 \\
\hline $\begin{array}{l}\text { Ucuzal M, Doğan R } \mathrm{R}^{(23) / \mathrm{Int}} \\
\text { Emerg Nurs/2015 }\end{array}$ & $\begin{array}{l}\text { Examinar o conhecimento, atitudes e habilidades dos } \\
\text { enfermeiros de emergência na dor }\end{array}$ & $\begin{array}{c}\text { Humanização, } \\
\text { Liderança }\end{array}$ & 5 \\
\hline $\begin{array}{l}\text { Pretorius A, et } \mathrm{al}^{(24)} / \text { Pain } \\
\text { Manag Nurs } / 2015\end{array}$ & $\begin{array}{l}\text { Identificar barreiras e facilitadores na enfermagem em } \\
\text { relação ao tratamento da dor }\end{array}$ & $\begin{array}{c}\text { Proatividade, } \\
\text { Desempenho assistencial, } \\
\text { Liderança }\end{array}$ & 4 \\
\hline $\begin{array}{l}\text { Cork LL }{ }^{(25)} / J \text { Trauma } \\
\text { Nurs/2014 }\end{array}$ & $\begin{array}{l}\text { Explorar a intuição dos enfermeiros em relação à gravidade } \\
\text { dos traumas nas emergências }\end{array}$ & $\begin{array}{c}\text { Liderança, } \\
\text { Trabalho em equipe }\end{array}$ & 5 \\
\hline $\begin{array}{l}\text { Fitzpatrick JJ, et al }{ }^{(26)} / \mathrm{J} \\
\text { Emerg Nurs } / 2014\end{array}$ & $\begin{array}{l}\text { Percepções do empoderamento dos enfermeiros e intenção } \\
\text { em abandonar a profissão }\end{array}$ & $\begin{array}{c}\text { Liderança, } \\
\text { Relacionamento interpessoal }\end{array}$ & 5 \\
\hline $\begin{array}{l}\text { Holanda FL, et } \mathrm{al}^{(27)} / \text { Acta } \\
\text { Paul Enferm/2015 }\end{array}$ & $\begin{array}{l}\text { Propor o perfil de competência profissional do enfermeiro } \\
\text { em emergências }\end{array}$ & $\begin{array}{l}\text { As oito competências elencadas } \\
\text { acima }\end{array}$ & 5 \\
\hline
\end{tabular}

Figura 3 - Sumário das características dos estudos sobre as competências do enfermeiro nas emergências. São Paulo, SP, Brasil, 2019

\section{Discussão}

As limitações deste estudo centram-se na possibilidade de alguma pesquisa não ter sido identificada no período de busca. Todavia avança no conhecimento ao identificar lacunas da literatura que precisam ser preenchidas, tais como o desenvolvimento de competências do enfermeiro em emergência e a relação destas com o produto do cuidar em enfermagem.

A atuação do enfermeiro em unidades de emergência pressupõe o desenvolvimento e a aplicabilidade de competências requeridas para atuação nesse espaço complexo e ao mesmo tempo singular, onde se exige do profissional um pensamento rápido e uma tomada de decisão acertada, baseada em conhecimen -tos prévios. As competências requeridas para o perfil desse profissional em emergência ${ }^{(27)}$ têm sua notoriedade em estudos, que, embora ainda incipientes, se propõem a contribuir e ao mesmo tempo construir um arcabouço teórico-prático das competências requeridas ao profissional que atua nesse ambiente.

As competências que mais emergiram nos artigos selecionados foram "desempenho assistencial" e "liderança". Em 70\% desses artigos ${ }^{(17,20-21,23-27)}$, foi realizado apenas o diagnóstico das competências mencionadas. Entretanto, há de se destacar que elas precisam ser desenvolvidas nos enfermeiros de emergência, de forma que tais competências norteiem suas 
condutas e esses profissionais sejam cada vez mais capacitados a prestar de forma crítica e fundamentada um cuidado que se traduza num produto do cuidar em enfermagem de alto patamar.

Os artigos que destacaram a competência "desempenho assistencial" ${ }^{\prime 17,22-23,25,27)}$ identificaram as condutas dos enfermeiros pautadas em conhecimento científico, dado o rigor de seu atendimento no serviço, apontando para um objetivo comum em relação à satisfação do paciente e direcionando suas ações para resultados que fossem traduzidos em qualidade do serviço prestado. Vale ressaltar que tal competência é definida como prestação de cuidados fundamentados em conhecimento científico, na qual o enfermeiro prestará uma assistência individualizada e que atenda às expectativas do cliente, a fim de gerar um resultado de qualidade ${ }^{(6)}$.

Outra competência encontrada nesta revisão foi a liderança ${ }^{(6)}$, cuja definição é o uso de estratégias que visem atingir o objetivo mediante a influência para o exercício de atividades diárias. Os estudos que destacaram a liderança enquanto competência $^{(23-24,26-27)}$ enfatizaram a capacidade de liderar do profissional enfermeiro nas unidades de emergência, em muitos momentos atuando à frente da equipe de enfermagem, multiprofissional e no gerenciamento do cuidado.

A liderança do enfermeiro nas unidades de emergência é importante para a implementação e desenvolvimento de ações que viabilizem a prestação de cuidados ao paciente. Esse profissional deve ter, em seu planejamento, as condições de previsão e provisão das atividades, pautando suas ações em uma liderança que articule as características individuais com as necessidades da organização ${ }^{(25,27)}$.

O serviço hospitalar de emergência requer uma equipe dinâmica e resolutiva que consiga atuar de forma sincronizada dentro das diversas situações, de forma que isso reflita no processo de recuperação e satisfação dos pacientes ${ }^{(27)}$. Sendo assim, a gestão do enfermeiro é uma atividade essencial e predominante, pois cabe a este executar as atividades de cuidado e gerenciar os recursos necessários proporcionando qualidade na entrega aos usuários ${ }^{(24)}$.

Em relação ao produto do cuidar em enfermagem, tem-se que múltiplos conhecimentos interferem nesse processo. Este saber específico do cuidar e do cuidado garantem ao enfermeiro a autonomia profissional na qual a competência reflete nas decisões to$\operatorname{madas}^{(18,27)}$.

Em sua evolução profissional, a enfermagem passou por diferentes fases em relação ao cuidado com o ser humano, o que lhe galgou construir um corpo de conhecimentos específicos proporcionando a construção da sua própria ciência ${ }^{(20)}$. 0 cuidado centrado nas reais necessidades do paciente tem sido concebido como um importante atributo para obter melhores resultados assistenciais e gerenciais. Esse modelo de concepção de cuidado é identificado como competência essencial dos enfermeiros ${ }^{(21,27)}$. Isso balizará uma (re)estruturação dos processos e um ambiente de trabalho favorável para o desempenho de tal comportamento. Portanto, é impossível dissociar o cuidado do processo que o produziu(14).

Nesse contexto, o produto do cuidar em enfermagem, avaliado a partir do instrumento Avaliação do Produto do Cuidar em Enfermagem ${ }^{(14)}$, proporcionará ao enfermeiro uma melhor dinâmica de cuidados, gerenciamento e resolutividade nas questões assistenciais e gerenciais. Portanto, sua aplicabilidade, em conjunto com as competências requeridas aos enfermeiros de emergência, norteará a assistência prestada ao paciente no atendimento de suas demandas.

Dessa forma, destaca-se maior aprimoramento do conceito do produto do cuidar em enfermagem por meio do instrumento Avaliação do Produto do Cuidar em Enfermagem ${ }^{(14)}$, principalmente por considerar que este ainda não foi testado na emergência. Essa escala é constituída por oito itens: planejamento da assistência de enfermagem; recursos necessários para prestar assistência; dimensionamento de pessoal de enfermagem; ações educativas e desenvolvimento de pessoal; acompanhamento e transferência do cuidado; interação e atuação multidisciplinar; atenção ao 
paciente e/ou familiar; e atendimento das necessidades assistenciais ${ }^{(14)}$.

As competências identificadas nos artigos desta revisão precisam ser desenvolvidas nos enfermeiros de emergência, de forma que elas norteiem suas condutas e eles sejam cada vez mais capacitados a prestar de forma crítica e fundamentada um cuidado que se traduza em produto do cuidar em enfermagem cada vez mais elevado.

\section{Conclusão}

As competências que mais emergiram foram desempenho assistencial e liderança. As produções sobre o produto do cuidado em enfermagem referem-se à criação e validação de um instrumento de medida, mas ainda não há estudos com a aplicabilidade desse conceito no contexto da emergência. Estudos futuros nessa linha poderão direcionar o enfermeiro de emergência a aperfeiçoar suas ações na perspectiva do produto do cuidar em enfermagem.

\section{Colaborações}

Jesus JA e Balsanelli AP colaboraram na concepção do projeto, análise e interpretação dos dados, redação do artigo e revisão crítica relevante do conteúdo intelectual, bem como na aprovação final da versão a ser publicada.

\section{Referências}

1. Mororó DDS, Enders BC, Lira ALBC, Silva CMB, Menezes RMP. Concept analysis of nursing care management in the hospital context. Acta Paul Enferm. 2017; 30(3):323-32. doi: https://doi. org/10.1590/1982-0194201700043

2. Santos JLG, Pestana AL, Higashi GDC, Oliveira RJT, Cassetari SSR, Erdmann AlL. Organizational context and care management by nurses at emergency care units. Rev Gaúcha Enferm. 2014; 35(4):5864.doi:http://dx.doi.org/10.1590/19831447.2014.04.45221
3. Kutney-Lee A, Mchugh MD, Sloane DM, Cimiotti JP, Flynn L, Neff DF, et al. Nursing: a key to patient satisfaction. Health Affairs. 2009; 28(4):669-77. doi: http://dx.doi.org/10.1377/hlthaff.28.4.w669

4. Santos JLG, Lima MADS, Pestana AL, Colomé ICS, Erdmann AL. Strategies used by nurses to promote teamwork in an emergency room. Rev Gaúcha Enferm. 2016; 37(1):e50178. doi: https:// doi.org/10.1590/1983-1447.2016.01.50178

5. Santos JLG, Pestana AL, Guerrero P, Meirelles BSH, Erdmann AL. Práticas de enfermeiros na gerência do cuidado em enfermagem e saúde: revisão integrativa. Rev Bras Enferm. 2013; 66(2):25763.doi:https://doi.org/10.1590/S003471672013000200016

6. Holanda FL, Marra CC, Cunha ICKO. Construction of a professional competency matrix of the nurse in emergency services. Acta Paul Enferm. 2014; 27(4):373-9. doi: http://dx.doi. org/10.1590/1982-0194201400062

7. Whittemore $\mathrm{R}$, Knafl $\mathrm{K}$. The integrative review: updated methodology. J Adv Nurs 2005; 52(5):546-53. doi: http://dx.doi.org/10.1111/ j.1365-2648.2005.03621.x

8. Mendes KDS, Silveira RCCP, Galvão CM. Integrative literature review: a research method to incorporate evidence in health care and nursing. Texto Contexto Enferm. 2008; 17(4):758-64. doi: http://dx. doi.org/10.1590/S0104-07072008000400018

9. Galvão TF, Pereira MG. Revisões sistemáticas da literatura: passos para sua elaboração. Epidemiol Serv Saúde. 2014; 23(1):183-4. doi: http://dx.doi. org/10.5123/S1679-49742014000100018

10. Cucolo DF, Perroca MG. Factors involved in the delivery of nursing care. Acta Paul Enferm 2015; 28(2):120-4. doi: dx.doi.org/10.1590/19820194201500021

11. Galvão CM, Sawada NO, Mendes IAC. A busca das melhores evidências. Rev Esc Enferm USP. 2003; 37(4):43-50. doi: http://dx.doi.org/10.1590/ S0080-62342003000400005

12. Galvão TF, Pansani TSA, Harrad D. Principais itens para relatar revisões sistemáticas e meta-análises: a recomendação PRISMA. Epidemol Serv Saúde. 2015; 24(2):335-42. doi: https://doi. org/10.5123/S1679-49742015000200017 
13. Cucolo DF, Perroca MG. Instrument to assess the nursing care product: development and content validation. Rev Latino-Am Enfermagem. 2015; 23(4):642-50. doi: http://dx.doi. org/10.1590/1518-8345.1495.2860

14. Cucolo DF, Perroca MG. Assessment of the nursing care product (APROCENF): a reliability and construct validity study. Rev Latino-Am Enfermagem. 2017; 25:e2860. doi: http://dx.doi. org/10.1590/1518-8345.1495.2860

15. Chitolli MR, Cucolo DF, Perroca MG. Assessment of the product of nursing care in specialized hospitals. Rev Bras Enferm. 2018; 71(6):2834-41. doi: http://dx.doi.org/10.1590/0034-7167-20170354

16. Rawson H, Bennett PN, Ockerby C, Hutchinson AM, Considine J. Emergency nurses' knowledge and self-rated practice skills when caring for older patients in the Emergency Department. Australas Emerg Nurs J. 2017; 20(4):174-80. doi: http:// dx.doi.org/10.1016/j.aenj.2017.08.001

17. Martínez-Segura E, Lleixà-Fortuño $\mathrm{M}$, Salvadó-Usach T, Solà-Miravete E, Adell-Lleixà $\mathrm{M}$, Chanovas-Borrás MR, et al. Competence of triage nurses in hospital emergency departments. Emergencias. 2017; 29(3):173-7. doi: http://dx.doi. org/10.1016/j.jen.2017.06.007

18. Jenkins J. On the night shift: advanced nurse practice in emergency medicine. Emerg Nurse. 2016; 24(2):22-6. doi: http://dx.doi.org/10.7748/ en.24.2.22.s24.23

19. Burchill CN, Polomano R. Certification in emergency nursing associated with vital signs attitudes and practices. Int Emerg Nurs 2016; 27:17-23. doi: http://dx.doi.org/10.1016/j.ienj.2015.12.003

20. Cabilan CJ, Eley R, Hughes JA, Sinnott M. Medication knowledge and willingness to nurse- initiate medications in an emergency department: a mixed-methods study. J Adv Nurs. 2016; 72(2):396-408. doi: http://dx.doi.org/10.1111/ jan.12840

21. Mello JJ, Bell JF, Siegel EO, Ward DH Evaluating psychiatric nursing competencies applied to emergency settings: a pilot role delineation study. Int Emerg Nurs. 2016; 25:37-42. doi: http://dx.doi. org/10.1016/j.ienj.2015.07.005

22. Thomas D, Kircher J, Plint AC, Fitzpatrick E, Newton AS, Rosychuk RJ, et al. Pediatric pain management in the emergency department: the triage nurses' perspective. J Emerg Nurs. 2015; 41(5):407-13. doi: http://dx.doi.org/10.1016/j. jen.2015.02.012

23. Ucuzal M, Doğan R. Emergency nurses' knowledge, attitude and clinical decision making skills about pain. Int Emerg Nurs. 2015; 23(2):75-80. doi: http://dx.doi.org/10.1016/j.ienj.2014.11.006

24. Pretorius A, Searle J, Marshall B. Barriers and enablers to emergency department nurses' management of patients' pain. Pain Manag Nurs. 2015; 16(3):372-9. doi: http://dx.doi.org/10.1016/j. pmn.2014.08.015

25. Cork LL. Nursing intuition as an assessment tool in predicting severity of injury in trauma patients. J Trauma Nurs. 2014; 21(5):244-52. doi: http:// dx.doi.org/10.1097/JTN.0000000000000072

26. Fitzpatrick JJ, Campo TM, Gacki-Smith J. Emergency care nurses: certification, empowerment, and work-related variables. J Emerg Nurs. 2014; 40(2):e37-43. doi: http://dx.doi.org/10.1016/j. jen.2013.01.021

27. Holanda FL, Marra CC, Cunha ICKO. Professional competency profile of nurses working in emergency services. Acta Paul Enferm. 2015; 28(4):308-14. doi: dx.doi.org/10.1590/1982-0194201500053

\section{(cc) BY}

Este é um artigo de acesso aberto distribuído sob os termos da Licença Creative Commons 